\title{
On the Minimal Ordered Morse Functions on Compact Simply-Connected Manifolds
}

By

Takao Matumoto*

\section{Introduction}

Throughout this paper, $\left(W^{n} ; V, V^{\prime}\right)$ denotes a compact smooth manifold triad of dimension $n$ in the sense that $\partial W=V \cup V^{\prime}$ and $V \cap V^{\prime}=\phi$, and $\mathscr{F}$ the space of $C^{\infty}$-functions of $\left(W ; V, V^{\prime}\right)$ into $(I ; 0,1)$ without critical points on the boundary. The codimension 0 stratum $\mathscr{F}^{0}$ of the natural stratification of $\mathscr{F}$ is the space of excellent functions (= Morse functions with distinct critical values). By definition, $f \in \mathscr{F}$ is called to be ordered and minimal, if the critical values are ordered in the order of their indices and the number of critical points is minimal with respect to the homology group structure of $H_{*}(W, V)$.

Let $f$ be an excellent function with $m$ critical values $v_{1}, \ldots, v_{n}$ and put $u_{0}=v_{1} / 2, u_{m}=1$ and $u_{i}=\left(v_{i}+v_{i+1}\right) / 2$ for $1 \leqq i \leqq m-1$. Then, $(W, V)$ has a filtration $W_{f}=\left(W_{0}, \ldots, W_{m}\right)$ defined by $W_{i}=f^{-1}\left[0, u_{i}\right]$ so that $W_{m}=W$ and $W_{i}$ has a presentation of the handle-attaching form $\left(W_{i-1}, f^{-1}\left(u_{i-1}\right) ; \varphi_{i} ; \lambda_{i}\right)$ in the notation of Smale [7]. For another filtration $\left(W_{0}^{\prime}, \ldots, W_{m}^{\prime}\right)$ with $W_{m}^{\prime}=W^{\prime}$, a map $h: W \rightarrow W^{\prime \prime}$ is called to be filtered if $h\left(W_{i}\right) \subset W_{\imath}^{\prime}$ for every $i$. A filtered map $h: W \rightarrow W^{\prime}$ is a filtered homotopy equivalence if there exists a filtered map $g: W^{\prime} \rightarrow W$ such that $(g \circ h) \mid W_{i}: W_{i} \rightarrow W_{i}$ and $(h \circ g) \mid W_{i}^{\prime}: W_{i}^{\prime} \rightarrow W_{i}^{\prime}$ are homotopy equivalences for every $i$.

We consider the following condition :

(*) $\pi_{1}(V)=\pi_{1}\left(V^{\prime}\right)=\pi_{1}(W, V)=\pi_{1}\left(W, V^{\prime}\right)=0, \operatorname{dim} W \geqq 6$ and $H_{*}(W$,

* Department of Mathematics, Kyoto University, Kyoto 606, Japan. 
V) has no torsions.

The purpose of this paper is to prove

Theorem. Suppose that (W,V) satisfies the condition (*). Then, two minimal ordered excellent functions $f$ and $f^{\prime}$ on $(W, V)$ belong to the same arcwise connected component of $\mathscr{F}^{0}$ if and only if the numbers of critical points are equal and there exists a filtered homotopy equivalence $h: W_{f}=\left(W_{0}, \ldots, W_{m}\right) \rightarrow W_{f^{\prime}}=\left(W_{0}^{\prime}, \ldots, W_{m}^{\prime}\right)$ such that $h \mid V=i d$ and $h \mid W_{m}: W \rightarrow W$ induces the identity on the homology group $H_{*}(W, V)$.

Corollary 1. Suppose the condition (*). Then, the space OM of minimal ordered Morse functions on $(W, V)$ is arcwise connected.

Definition. Let $f \in \mathscr{F}$. An element $h$ of $\operatorname{Diff}(W, V ; f)$ is a diffeomorphism of $W$ such that $h \mid V=i d$ and $h$ preserves the level of $f$, i. e. : $f \circ h=f$. A diffeomorphism $g$ of $(W, V)$ is called a special diffeomorphism if $g \mid V=i d$ and the induced map $g_{*}: H_{*}(W, V) \rightarrow$ $H_{*}(W, V)$ is the identity.

Corollary 2. Let $f$ be a minimal ordered excellent function on $(W, V)$. Suppose the condition (*). Then, the natural homomorphism of the group $\pi_{0}(D i f f(W, V ; f))$ of the isotopy classes of level-preserving diffeomorphisms into the group $\pi_{0}(\operatorname{SDiff}(W, V))$ of the isotopy classes of special diffeomorphisms of $(W, V)$ is a surjection.

The theorem above is a generalization of the theorem of connectivity of the space of functions without critical points established by Cerf [2]. Corollary 1 implies that two ordered minimal handle-decomposition on such a manifold become isotopic after some repeat of reorderings and handle-adding operations; Agoston [1] has proved a weaker version of the uniqueness of the minimal handle-decomposition up to isotopy by using only the $h$-cobordism theorem, and he gave an example which showed that the torsion-freeness was indispensable to his 
uniqueness theorem and consequently to Corollary 1 . We shall give another stronger counter-example, that is, an example of non-diffeomorphic minimal handle-decompositions in the appendix $(=\S 4)$. It is not difficult to find an example which shows the failure of the theorem when $H_{*}(W, V)$ has some torsion elements. In fact, I acknowledge that Professor F. Laudenbach wrote me such an example. Corollary 2 would be useful in the study of $\pi_{0}(\operatorname{Diff}(W, V))$.

\section{§1. Proof of Theorem}

Assume that $f_{t}, 0 \leqq t \leqq 1$, is a smoothly parametrized family of excellent functions on $(W, V)$ such that $f_{0}=f$ and $f_{1}=f^{\prime}$. Then, because the critical points are smoothly parametrized, the usual construction of the gradient-like vector field using a partition of unity gives a smoothly parametrized family of vector fields $\xi_{t}, 0 \leqq t \leqq 1$, so that every $\xi_{t}$ is a gradient-like vector field for $f_{t}$. Let $W_{i, t}$ denote the $i$-th filtration associated to $f_{t}$ and $\partial_{+} W_{i, t}=\left(f_{t}\right)^{-1}\left(u_{i, t}\right)$. Then, because $[0,1]$ is compact, there exists a sequence $0=t(0)<t(1)<\ldots<t(\ell)=1$ such that the flows associated to $\xi_{t}$ with $t=t(j)$ are transverse with $\partial_{+} W_{i, t}$ for every $i$ and $t \in[t(j), t(j+1)]$. Therefore, by sliding along the flows, we get easily an isotopy $\left(h_{j}\right)_{s}, 0 \leqq s \leqq 1$, of diffeomorphisms of $(W, V)$ such that $\left(h_{j}\right)_{0}=i d,\left(h_{j}\right)_{s} \mid V=i d$ and $\left(h_{j}\right)_{1}\left(W_{i, t(j)}\right)=W_{i, t(j+1)}$. If we compose these $\ell$ isotopies, we get an isotopy $h_{t}, 0 \leqq t \leqq 1$, such that $h_{0}=i d, h_{t} \mid V=i d$ and $h_{1}\left(W_{i, 0}\right)=W_{i, 1}$. Clearly $h_{1}$ is a required filtered homotopy equivalence.

Now we shall prove the sufficiency of the filtered homotopy equivalence condition: Let $f$ and $f^{\prime}$ be two minimal ordered excellent functions on $(W, V)$. Composing a diffeomorphism of $I=[0,1]$ onto $[-1, m]$ and adjusting the critical values of excellent functions, we may assume that the critical values of $f$ and $f^{\prime}$ are $0,1,2,3, \ldots, m-1$. First of all, we note that, for any isotopy $h_{t}$ of $W, f_{\supset} h_{t}$ is a family of excellent functions that is, a path in $\mathscr{F}^{0}$.

If $V=\phi$, it is easy to get an isotopy $h_{t}, 0 \leqq t \leqq 2$, of $W$ such that $f^{\prime} \circ h_{2}$ coincides with $f$ on $f^{-1}[0,1 / 3]:$ in fact, since $\pi_{1}(W, V)=0$, each of the minimal functions $f$ and $f^{\prime}$ has a unique critical point 
of index 0 , which is denoted by $p_{0}$ or $p_{0}^{\prime}$ respectively. Hence, an isotopy $h_{t}, 0 \leqq t \leqq 1$, can satisfy that $h_{0}=i d$ and $h_{1}\left(p_{0}\right)=p_{0}^{\prime}$. Then, the uniqueness of the disk neighborhood implies $h_{1}\left(f^{-1}[0,1 / 3]\right)=$ $\left(f^{\prime}\right)^{-1}[0,1 / 3]$ and there is an isotopy $h_{t}, 1 \leqq t \leqq 2$, of $W$ such that $f=f^{\prime} \circ h_{2}$ on $f^{-1}[0,1 / 3]$. Therefore, we have only to compare $f$ and $f^{\prime} \circ h_{2}$ on the manifold $f^{-1}[1 / 3, m]$ and we may assume $f^{-1}(1 / 3) \neq \phi$. If $V \neq \phi$, we may assume that $f$ and $f^{\prime}$ coincide on $f^{-1}[-1,-2 / 3]$. Remark that $f$ and $f^{\prime}$ have no critical points of index 1 , because $\pi_{1}(W, V)=0$.

Therefore, in both cases when $V=\phi$ or $V \neq \phi$, we may assume as a hypothesis of induction that the minimal excellent functions $f$ and $f^{\prime}$ coincide on $f^{-1}(-\infty, i-2 / 3]$ and the filtered homotopy equivalence $h$ satisfies $h \mid f^{-1}(-\infty, i-2 / 3]=i d$. Then, $h$ must induce the identify on $H_{*}\left(\bar{W}, V_{i}\right)$ by the minimality of $f$ and the torsion-freeness of $H_{*}(W, V), \bar{W}$ and $V_{\text {, denoting }} f^{-1}[i-2 / 3, m]$ and $f^{-1}(i-2 / 3)$ respectively. Hence, we have reduced the problem to compare the restricted minimal excellent functions $f$ and $f^{\prime}:\left(\bar{W} ; V_{i}, V^{\prime}\right) \rightarrow$ $([i-2 / 3, m] ; i-2 / 3, m)$ which have no critical points of index less than $k$ with $2 \leqq k \leqq n-3$. Let $p$ be the lowest critical point of index $k$ of $f$. Choosing a gradient-like vector field $\xi$ for $f$, we define a left-hand disk $D_{L}(p)$ whose boundary $S_{L}(p)$ is contained in $V_{i}$, by the union of segments of integral curves of $\xi$ beginning at $p ; D_{L}(p)$ represents an element of $\pi_{k}\left(\bar{W}, V_{i}\right)=H_{k}\left(\bar{W}, V_{i}\right)$ [6].

Let $w_{i+1}$ and $w_{i+1}^{\prime}$ denote the elements of $H_{k}\left(\bar{W}, V_{i}\right)$ represented by $W_{i+1} \cap \bar{W}$ and $W_{i+1}^{\prime} \cap \bar{W}$ respectively. Then, we have $(h \mid \bar{W})_{*} r w_{i+1}=$ $w_{i+1}^{\prime}$; and since $(h \mid \bar{W})_{*}=i d, w_{i+1}=w_{i+1}^{\prime}$. Moreover, because $V_{i} \cup D_{L}(p)$ and $V_{i} \cup D_{L}^{\prime}\left(p^{\prime}\right)$ are the deformation retracts of $W_{i+1} \cap \bar{W}$ and $W_{i+1}^{\prime} \cap \bar{W}$ respectively in $\left(\bar{W}, V_{i}\right)$, we see that $D_{L}(p)$ and $D_{L}^{\prime}\left(p^{\prime}\right)$ represent the same element of $\pi_{k}\left(\bar{W}, V_{i}\right)$. Since we restrict ourselves to the case in which $k \leqq n-3$ and $\pi_{s}\left(\bar{W}, V_{i}\right)=0$ for $j \leqq k-1$, we can apply the theorem of uniqueness of the handle-attaching due to Hudson [5; Corollary 2.1] in order to get an isotopy $h_{t}, 0 \leqq t \leqq 1$, of $\bar{W}$ starting from the identity $h_{0}$ such that $h_{1}\left(D_{L}(p)\right)=D_{L}^{\prime}\left(p^{\prime}\right)$; moreover, composing an extension of an isotopy of $D_{L}(p)$, we may assume that $h_{1}(p)=p^{\prime}$. 
Note that we have an isotopy $g_{t}, 0 \leqq t \leqq 1$, of $D_{L}^{\prime}\left(p^{\prime}\right)$ such that $g_{0}=i d$ and $f^{\prime} \circ g_{1} \circ h_{1}=f$ on a disk neighborhood of $p$ in $D_{L}(p)$; we may assume that this neighborhood has the form of $f^{-1}[i-\varepsilon, i] \cap D_{L}(p)$ for some small positive $\varepsilon$ and that the isotopy $g_{t}$ is extended on $\bar{W}$. It is not difficult to choose an extension $g_{t}$ so that two smooth collar neighborhoods $\left(g_{1} \circ h_{1}\right)\left(f^{-1}[i-2 / 3, i-\varepsilon]\right)$ and $\left(f^{\prime}\right)^{-1}[i-2 / 3, i-\varepsilon]$ of $V_{i}$ in $\bar{W}$ coincide with each other in a neighborhood of $D_{L}^{\prime}\left(p^{\prime}\right) \cap$ $\left(f^{\prime}\right)^{-1}[i-2 / 3, i-\varepsilon]$. Moreover, by the uniqueness of the smooth collar neighborhood, we have an isotopy $g_{t}, 1 \leqq t \leqq 2$, of $\bar{W}$ such that $\left(g_{2} \circ h_{1}\right)$ $\left.\left(f^{-1}[i-2 / 3, i-\varepsilon]\right)=\left(f^{\prime}\right)^{-1}[i-2 / 3, i-\varepsilon],\left(g_{2} \circ h_{1}\right)\left(D_{L}(p) \cap f^{-1}[i-\varepsilon, i]\right)\right)=$ $D_{L}^{\prime}\left(p^{\prime}\right) \cap\left(f^{\prime}\right)^{-1}[i-\varepsilon, i]$ and $f^{\prime} \circ g_{2} \circ h_{1}=f$ on $f^{-1}[i-\varepsilon, i] \cap D_{L}(p)$. Then, because $f$ and $f^{\prime} \circ g_{2} \circ h_{1}$ have no critical points on $f^{-1}[i-2 / 3, i-\varepsilon]$, we can apply the theorem of Cerf [2] to deform $f^{\prime} \circ g_{2} \circ h_{1}$ by a path $f_{t}^{\prime}$ in $\mathscr{F}^{0}$ such that $f_{0}^{\prime}=f^{\prime} \circ g_{2} \circ h_{1}, f_{1}^{\prime}=f$ on $f^{-1}[i-2 / 3, i-\varepsilon]$ and that for every $t, f_{t}^{\prime}=f^{\prime} \circ g_{2} \circ h_{1}$ on $f^{-1}[i-\varepsilon, \infty)$. Hence, we have only to consider the manifold $f^{-1}[i-\varepsilon, \infty)$. Let $m:[i-\varepsilon, \infty) \rightarrow[i-2 / 3, \infty)$ be a smooth map free from critical points and with $m \mid[i, \infty)=i d$. We rewrite $\bar{W}, V_{i}, f, f^{\prime}, D_{L}(p)$ and $D_{L}^{\prime}\left(p^{\prime}\right)$ instead of $f^{-1}[i-\varepsilon, \infty)$, $f^{-1}(i-\varepsilon), m \circ f\left|f^{-1}[i-\varepsilon, \infty), m \circ f^{\prime} \circ g_{2} \circ f_{1}\right| f^{-1}[i-\varepsilon, \infty), D_{L}(p) \cap f^{-1}[i-\varepsilon, i]$ and $D_{L}^{\prime}\left(p^{\prime}\right) \cap\left(f_{0}^{\prime} \circ g_{2} \circ h_{1}\right)^{-1}[i-\varepsilon, i]$ respectively. Then, we have $p=p^{\prime}$, $D_{L}^{\prime}\left(p^{\prime}\right)=D_{L}(p)$ and $f=f^{\prime}$ on $D_{L}^{\prime}\left(p^{\prime}\right)=D_{L}(p)$.

Now we may assume that $N(p)=f^{-1}[i-2 / 3, i+1 / 3]$ and $N\left(p^{\prime}\right)=$ $\left(f^{\prime}\right)^{-1}[i-2 / 3, i+1 / 3]$ are the smooth regular neighborhoods of $D_{L}(p) \cup V$. Taking a small disk-neighborhood $D$ of $p=p^{\prime}$ in $N(p) \cap$ $N^{\prime}\left(p^{\prime}\right)$, we consider two handles $H(p)$ and $H^{\prime}\left(p^{\prime}\right)$ which are defined by the union of segments contained in $N(p)$ and $N^{\prime}\left(p^{\prime}\right)$ respectively, of the integral curves of $\pm h_{1}(\xi)$ and $\pm \xi^{\prime}$ respectively beginning at some points of $D$. Because $H(p)$ and $H^{\prime}\left(p^{\prime}\right)$ are the smooth regular neighborhoods of $D_{L}(p)=D_{L}^{\prime}\left(p^{\prime}\right)$, we have an isotopy $h_{t}, 0 \leqq t \leqq 1$, of $\bar{W}$ starting from the identity $h_{0}$ such that $h_{1}(H(p))=H^{\prime}\left(p^{\prime}\right)$ and $h_{1} \mid D_{L}(p)=i d$. It is not difficult to assume $h_{1}(N(p))=N^{\prime}\left(p^{\prime}\right)$. On the other hand, there are diffeomorphisms $g$ and $g^{\prime}$ of $h_{1}(H(p))=H^{\prime}\left(p^{\prime}\right)$ onto the standard handle $D^{k, n-k}=\left\{\left(x_{1}, \ldots, x_{k}, y_{1}, \ldots, y_{n-k}\right) \in D^{k} \times D^{n-k}\right.$; $\left.\left(x_{1}^{2}+\ldots+x_{k}^{2}\right)\left(y_{1}^{2}+\ldots+y_{n-k}^{2}\right) \leqq 1\right\}$, which map each level submanifold onto some level submanifold and restrict to the same diffeomorphism 
of $D_{L}(p)=D_{L}^{\prime}\left(p^{\prime}\right)$ onto $D^{k} \times 0$.

Lemma 1. Let $f_{0}$ be a standard Morse function on $D^{p} \times D^{q}$, i. e. : $f_{0}=x_{1}^{2}+\ldots+x_{p}^{2}-y_{1}^{2}-\ldots-y_{q}^{2}$. Then, any auto-diffeomorphism of $D^{p q}=\left\{\left(x_{1}, \ldots, x_{p}, y_{1}, \ldots, y_{q}\right) \in D^{p} \times D^{q} ;\left(x_{1}^{2}+\ldots+x_{p}^{2}\right)\left(y_{1}^{2}+\ldots+y_{q}^{2}\right) \leqq 1\right\}$, which restricts to the identity on $D^{p} \times 0 \cup \partial_{-} D^{p, q}$, is isotopic by a path of such auto-diffeomorphisms to a diffeomorphism $h$ such that $f_{0} \circ h=f_{0}$, where $\partial_{-} D^{p, q}$ denotes $D^{p, q} \cap \partial D^{p} \times D^{q}$.

Proof. Let $\alpha: D^{p} \rightarrow S O(q)$ be a differentiable map into the rotation group satisfying $\alpha \mid \partial D^{p}=1$. Then, the automorphism $d(\alpha)=h$ of $D^{p, q}$ defined by $h(x, y)=(x, \alpha(x) y)$ gives a diffeomorphism of $D^{p, q}$ relative to $D^{p} \times 0 \cup \partial_{-} D^{p, q}$. As a consequence, we get a homomorphism $d: \pi_{p}(S O(q)) \rightarrow \pi_{0}\left(\operatorname{Diff}\left(D^{p, q}, D^{p} \times 0 \cup \partial_{-} D^{p, q}\right)\right.$. It is standard in the tubular neighborhood theory to see that $d$ is an isomorphism (cf. [4]). So, any auto-diffeomorphism of $D^{p, q}$ relative to $D^{p} \times 0 \cup$ $\partial_{-} D^{p, q}$ has a representative of the form $h(x, y)=(x, \alpha(x) y)$ in the isotopy class, and evidently this diffeomorphism satisfies that $f_{0} \circ h=f_{0}$.

q. e. d.

Since $g^{\prime} \circ g^{-1}$ is a diffeomorphism of $D^{k, n-k}$ relative to $D^{k} \times 0 \cup \partial_{-} D^{k, n-k}$, it is isotopic by a path consisting of a family of diffeomorphisms $g_{t}$, $0 \leqq t \leqq 1$, to a level-preserving diffeomorphism $g_{1}$ by Lemma 1 . Hence, $\left(g^{\prime}\right)^{-1} \circ g_{1} \circ g$ is a level-preserving diffeomorphism of $h_{1}(H(p))$ onto $H^{\prime}\left(p^{\prime}\right)$. By composing some monotone map of $[i-2 / 3, i+1 / 3]$ onto $[i-2 / 3, i+1 / 3]$, we may assume that $f^{\prime} \circ\left(g^{\prime}\right)^{-1} \circ g_{1} \circ g=f \circ h_{1}^{-1}$ on $h_{1}(H(p))$. The isotopy of embeddings $\left(g^{\prime}\right)^{-1} \circ g_{t} \circ g, 0 \leqq t \leqq 1$, of $h_{1}(H(p))$ can be extended to an isotopy of embeddings of $N(p)$ and then to an isotopy $h_{t}, 1 \leqq t \leqq 2$, of $\bar{W}$. Therefore, $f^{\prime} \circ h_{t}, 0 \leqq t \leqq 2$, is a path in $\mathscr{F}^{0}$ such that $f^{\prime} \circ h_{2}=f$ on $H(p)$; moreover, because each of $f^{\prime} \circ h_{2}$ and $f$ has no critical points on $N(p)-H(p)$, these two functions can be connected by a path of functions which have no critical points on $N(p)-H(p)$ by the theorem of Cerf [2]. As a consequence, $f^{\prime}$ can be deformed by a path in $\mathscr{F}^{0}$ into a $f_{1}^{\prime}$ such that $f_{1}^{\prime}=f$ on $f^{-1}(-\infty, i+1 / 3]$. 
If we consider the manifold $f^{-1}[i+1 / 3, \infty)$, we have diminished the number of the critical points of index $k$ with $k \leqq n-3$; Therefore, by the induction on the number of the critical points of index $k$ with $k \leqq n-3$, we can obtain a path $f_{t}^{\prime}$ in $\mathscr{F}^{0}$ such that $f_{1}^{\prime}=f$ on $f^{-1}\left(-\infty, i_{0}+1 / 3\right]$ and $f_{0}^{\prime}=f^{\prime}$, assuming that $i_{0}$ is the heighest critical value of the index $k_{0}$ where $n=k_{0}+3$.

If we consider the minimal ordered excellent functions $m-1-f$ and $m-1-f^{\prime}$, we may assume in the same way that $f_{1}^{\prime}=f$ on $f^{-1}\left[i_{0}+2 / 3, \infty\right)$, because $n-k_{0}=3 \leqq n-3$. Moreover, since $f$ and $f_{1}^{\prime}$ have no critical points on $f^{-1}\left[i_{0}+1 / 3, i_{0}+2 / 3\right]$, they can be connected by a path consisting of the functions without critical points on $f^{-1}\left[i_{0}+1 / 3, i_{0}+2 / 3\right]$ by the theorem of Cerf [2]. Hence, original $f$ and $f^{\prime}$ are connected by a path in $\mathscr{F}^{0}$; this completes the proof of Theorem.

Remark. If we review the proof, we have connected $f$ and $f^{\prime}$ only by the following three types of paths in $\mathscr{F}^{0}$ : type $(1)=\mathrm{a}$ path given by adjusting the critical values, type $(2)=a$ path of the form $f \circ h_{t}$ where $h_{t}$ is an isotopy of $W$ (in fact, we mentioned only on the isotopy defined on the submanifold of codimension 0 but it is easily extended on $W$ ), and type (3) =a path given by the theorem of Cerf ; we can easily reduce this case to the path of type (2) by using a smoothly parametrized vector fields. Hence, if $f$ and $f^{\prime}$ have the same critical values from the beginning, we do not need the path of type (1) and we get an isotopy $h_{t}$ of $W$ such that $f=f^{\prime} \circ h_{1}$. If we modify the isotopy in the collar neighborhood of $V$, we may assume that this isotopy $h_{t}$ satisfies $h_{t} \mid V=i d$.

\section{§2. Proof of Corollary 1}

Let $f$ and $f^{\prime}$ be two minimal ordered Morse functions on $(W, V)$. Composing a diffeomorphism of $I$ onto $[-1 / 2, n+1 / 2]$ and adjusting the critical values of Morse functions, we may assume that $f$ and $f^{\prime}$ are minimal nice functions, i. e.: the critical values of index $k$ is equal to $k$. 
By the argument in the proof of Theorem, it is sufficient to prove that there exists a path $f_{t}, 0 \leqq t \leqq 1$, in $\mathcal{O} \mathscr{M}$ such that $f=f_{0}$ on $f^{-1}(-\infty, k+1 / 3]$ and $f^{\prime}=f_{1}$, assuming that $f$ and $f^{\prime}$ have no critical points of index less than $k$ with $2 \leqq k \leqq n-3$ and $f(V)=f^{\prime}(V)=k-2 / 3$. Now, let $p_{1}, \ldots, p_{j}$ be the critical points of index $k$ of $f_{\text {. Choosing a }}$ gradient-like vector-field $\xi$ for $f$, we define left-hand disks $D_{L}\left(p_{1}\right), \ldots$, $D_{L}\left(p_{j}\right)$ whose boundaries are contained in $V$ so that they represent the elements in $\pi_{k}(W, V)=H_{k}(W, V)$.

Lemma 2. Suppose that $2 \leqq k \leqq n-2$ and $\pi_{k}(W, V)$ has no torsions. Then, there is a path $f_{t}^{\prime}, 0 \leqq t \leqq 1$, in $\mathcal{O} \mathscr{M}$ with $f_{1}^{\prime}=f^{\prime}$ which satisfies the following property: there exists a gradient-like vector field $\xi^{\prime}$ for $f_{0}^{\prime}$ such that for each $i$ with $1 \leqq i \leqq j$ the left-hand disk $D_{L}^{\prime}\left(p_{i}^{\prime}\right)$ associated to $f_{0}^{\prime}$ and $\xi^{\prime}$ represents the same element of $\pi_{k}(W, V)$ as $D_{L}\left(p_{i}\right)$, where $p_{1}^{\prime}, \ldots, p_{j}^{\prime}$ denote the critical points of index $k$ of $f_{0}^{\prime}$.

Proof. Since $H_{k}(W, V)$ has no torsions, the natural map $H_{k}\left(\left(f^{\prime}\right)^{-1}[k-2 / 3, k+1 / 3], V\right) \rightarrow H_{k}(W, V)\left(=\pi_{k}(W, V)\right)$ is an isomorphism. So, applying the basis theorem 6.7 of [6], we obtain a Morse function $f_{0}^{\prime}$ on $\left(f^{\prime}\right)^{-1}[k-2 / 3, k+1 / 3]$ and a gradient-like vector field $\xi^{\prime}$ for $f_{0}^{\prime}$ such that the family of $D_{L}^{\prime}\left(p_{i}^{\prime}\right), 1 \leqq i \leqq j$, constitutes the corresponding basis in $H_{k}\left(\left(f^{\prime}\right)^{-1}[k-2 / 3, k+1 / 3], V\right)$. Actually, this $f_{0}^{\prime}$ is constructed by a successive use of the following procedure: increasing $f^{\prime}$ in the neighborhoods of some critical points and then readjusting the Morse function so that there is only one critical value of index $k$. This implies that $f^{\prime}$ and $f_{0}^{\prime}$ can be connected by a path in $\mathcal{O} \mathscr{M}$.

q. e. d.

Therefore, by using the theorem of Hudson on the uniqueness of the handle-attaching as in the proof of Theorem, we can get an isotopy $h_{t}, 0 \leqq t \leqq 1$, of $(W, V)$ such that $h_{0}=i d, h_{t} \mid V=i d$ and $f=f_{0}^{\prime} \circ h_{1}$ on $f^{-1}(-\infty, k+1 / 3]$. By the remark preceding Lemma 2, this is enough to get a path $f_{t}, 0 \leqq t \leqq 1$, in $\mathcal{O} \mathscr{M}$ such that $f_{0}=f$ and $f_{1}=f^{\prime}$. 


\section{§. Proof of Corollary 2}

Let $f$ be a minimal ordered excellent function on $(W, V)$ and let $h$ be a special diffeomorphism of $(W, V)$. We shall show that there is an isotopy $h_{t}, 0 \leqq t \leqq 1$, of diffeomorphism of $(W, V)$ such that $h_{0}=i d, h_{t} \mid V=i d$ and $f \circ\left(h \circ h_{1}\right)=f$. We observe first that $h^{-1}$ is a filtered homotopy equivalence of $W_{f}$ into $W_{f \circ h}$ which induces the identity on $H_{*}(W, V)$. Then, by our Theorem, $f \circ h$ and $f$ can be connected by a path in $\mathscr{F}^{0}$, moreover, by the remark at the end of $\S 1$, there exists an isotopy $h_{t}, 0 \leqq t \leqq 1$, of the diffeomorphisms of $(W, V)$ such that $h_{0}=i d, h_{t} \mid V=i d$ and $f=(f \circ h) \circ h_{1}=f \circ\left(h \circ h_{1}\right)$.

\section{§4. Appendix : Non-diffeomorphic minimal handle-decompositions}

We shall show

Proposition. There are two different minimal nice functions $f$ and $f^{\prime}$ on a 5-connected 13-manifold $W$ with $V^{\prime}=\partial W=S^{12}$ and $H_{6}(W)=Z / 13 \oplus Z / 13$ such that there is no diffeomorphism $h$ of $W$ satisfying $h\left(\left(f^{\prime}\right)^{-1}(6+1 / 2)\right)=f^{-1}(6+1 / 2)$.

Agoston's example [1] shows that some diffeomorphic minimal handle-decompositions cannot be isotopic; our example concerns, however, the ones which cannot be mutually diffeomorphic.

Let $X_{0}$ be the boundary connected sum $S^{6} \times D^{7}$ म $S^{6} \times D^{7}$ and $X_{1}$ be $X_{0}-\operatorname{int} D^{13}$. We shall construct two auto-diffeomorphisms $h$ and $h^{\prime}$ of $\partial X_{0}=S^{6} \times S^{6} \# S^{6} \times S^{6}$ so that $W=X_{0} \cup_{h} X_{1}$ and $W^{\prime}=X_{0} \cup_{h^{\prime}} X_{1}$ are mutually diffeomorphic but there is no diffeomorphism $H: W \rightarrow W^{\prime}$ satisfying $H\left(X_{0}\right)=X_{0}$. Let $u_{1}$ and $u_{2}$ be the generators of $H_{6}\left(S^{6} \times S^{6} \# S^{6} \times S^{6}\right)$ corresponding the natural embeddings of $S^{6}$ onto $S^{6} \times 0$ in the first connected sum component and in the second component respectively. Define $v_{1}$ and $v_{2}$ similarly by using $0 \times S^{6}$ instead. Then, any autodiffeomorphism $h$ of $\partial X_{0}$ induces a matrix $\mu(h)=M$ which is defined 
by $\left(h_{*} u_{1}, h_{*} u_{2}, h_{*} v_{1}, h_{*} v_{2}\right)^{*}=M\left(u_{1}, u_{2}, v_{1}, v_{2}\right)^{*}$, where * stands for the transpose.

Lemma 3. The anti-homomorphism $\mu: \operatorname{Diff}\left(\partial X_{0}\right) \rightarrow G L(4, Z)$ maps onto the group $O(4, Z)$ of all $4 \times 4$-matrices of integers $M$ which satisfy the condition:

$$
M J M^{*}= \pm J \text {, where } J=\left(\begin{array}{ll}
0 & I \\
I & 0
\end{array}\right) .
$$

For the proof, we refer the paper of Wall on the diffeomorphisms of 4-manifolds [11]: in fact, reading $N=S^{6} \times S^{6}$ and $S=S^{6} \times S^{6}$, we have no difficulties to verify this Lemma.

Let $\mu(h)=\left(\begin{array}{ll}R & S \\ P & Q\end{array}\right)$ and $\mu\left(h^{\prime}\right)=\left(\begin{array}{ll}R^{\prime} & S^{\prime} \\ P^{\prime} & Q^{\prime}\end{array}\right)$ are the matrices represented by $2 \times 2$-matrix blocks.

Lemma 4. Suppose that $P^{\prime}=P$. Then, $W=X_{0} \cup_{h} X_{1}$ is diffeomorphic to $W^{\prime}=X_{0} \cup_{h^{\prime}} X_{1}$.

Proof. Since any embedded 6-sphere in $X_{0}$ has a trivial normal bundle and the natural map $H_{6}\left(X_{0}\right) \rightarrow H_{6}(W)$ is onto, the Wall's invariant $\alpha: H_{6}(W) \rightarrow \pi_{5}(S O)$ of the almost closed 5-connected 13manifold $W$ is zero [12]. So, by Theorem 8 of Wall [loc. cit], we have a handlebody $L \in \mathscr{H}(14, k, 7)$ such that $W=\partial L-\operatorname{int} D^{13}$. In the same way, we have another handlebody $L^{\prime} \in \mathscr{H}\left(14, k^{\prime}, 7\right)$ such that $W^{\prime}=\partial L^{\prime}-\operatorname{int} D^{13}$. Moreover, $P=P^{\prime}$ implies that $H_{6}(W)=H_{6}\left(W^{\prime}\right)$ and hence $H_{6}(\partial L)=H_{6}\left(\partial L^{\prime}\right)$. According to Theorem 6.3 of Smale [10], $\partial L^{\prime}$ is diffeomorphic to $\partial L^{\prime}$ and, in particular, $W$ is diffeomorphic to $W^{\prime}$.

q. e. d.

Lemma 5. Suppose that $P=P^{\prime}$ and the elementary divisors of $P$ are not all zero. If there exists a diffeomorphism $H: W \rightarrow W^{\prime}$ satisfying $H\left(X_{0}\right)=X_{0}$, then 
$\operatorname{det} R \equiv \operatorname{det} R^{\prime}\left(\bmod p_{1}\right)$ and $\operatorname{det} Q \equiv \operatorname{det} Q^{\prime}\left(\bmod p_{1}\right)$,

where $p_{1}$ denotes the first elementary divisor of $P$.

The proof of this lemma is similar to that of Theorem 2 of Birman [9]; we have only to replace her $J$ with our $J$ in Lemma 3 and the symmetric matrices with skew-symmetric matrices.

Now, we are in a position to prove Proposition. Since the following two matrices

$$
M=\left(\begin{array}{rrrr}
0 & 4 & 1 & 0 \\
-4 & 0 & 0 & 1 \\
13 & 0 & 0 & -3 \\
0 & 13 & 3 & 0
\end{array}\right) \text { and } M^{\prime}=\left(\begin{array}{rrrr}
0 & 2 & 1 & 0 \\
-2 & 0 & 0 & 1 \\
13 & 0 & 0 & -6 \\
0 & 13 & 6 & 0
\end{array}\right)
$$

are the elements of the orthogonal group $O(4, \mathbb{Z})$, we have two auto-diffeomorphisms $h$ and $h^{\prime}$ of $\partial X_{0}$ such that $\mu(h)=M$ and $\mu\left(h^{\prime}\right)=M^{\prime}$ by Lemma 3. Then, $W=X_{0} \cup_{h} X_{1}$ and $W^{\prime}=X_{0} \cup_{h^{\prime}} X_{1}$ are mutually diffeomorphic by Lemma 4 ; in fact, $H_{6}(W)=Z / 13 \oplus Z / 13$. We cannot, however, obtain any diffeomorphism $H: W \rightarrow W^{\prime}$ satisfying $H\left(X_{0}\right)=X_{0}$ according to Lemma 5. Because $W$ has a minimal nice function $f$ with $f^{-1}(-\infty, 6+1 / 2]=X_{0}$ and $W^{\prime}$ has a $f^{\prime}$ with $\left(f^{\prime}\right)^{-1}(-\infty, 6+1 / 2]=X_{0}$, this completes the proof of Proposition.

We can remark that the same proof can apply to obtain a 1connected 5-manifold $W$ with $w_{2}(W)=0$ and $H_{2}(W)=\mathbb{Z} / 13 \oplus Z / 13$ which admits two different minimal nice functions $f$ and $f^{\prime}$ such that there is no diffeomorphism $h$ of $W$ satisfying $h\left(\left(f^{\prime}\right)^{-1}(2+1 / 2)\right)=$ $f^{-1}(2+1 / 2)$.

\section{References}

[1] Agoston, M.K., On handle decompositions and diffeomorphisms, Trans. Amer. Math. Soc., 137 (1969), 21-26.

[2] Cerf, J., La stratification naturelle des espaces de fonctions différentiables reélles et le théorème de la pseudo-isotopie. Publ. Math.I.H.E.S., 39(1970), 5-173.

[3] Haefliger, A., Plongements différentiables des variétés dans variétés, Comment. Math. Helv., 36 (1962), 47-82. 
[4] Hirsch, M.W., Differential Topology, Springer-Verlag, 1976.

[5] Hudson, J. F. P., Embeddings of bounded manifolds, Proc. Camb. Phil. Soc., 72 (1972), 11-20.

[6] Milnor, J., Lectures on the h-cobordism theorem, Princeton Univ. Press, 1965.

[7] Smale, S., Generalized Poincaré's conjecture in dimensions greater than four, Ann. of Math., 74 (1961), 391-406.

[8] On the structure of manifolds, Amer.J.Math., 84 (1962), 387-399.

\section{References added for Appendix}

[9] Birman, J.S., On the equivalence of Heegaard splittings of closed, orientable 3-manifolds, Annals of Math. Studies no. 84 edited by Neuwirth (1975), 137-164.

[10] Smale, S., On the structure of 5-manifolds, Ann. of Math., 75 (1963), 38-46.

[11] Wall, C. T.C., Diffeomorphisms of 4-manifolds, J. London Math. Soc., 39 (1964), 131140.

[12] Classification problems in differential topology-VI, classification of (s-1)connected (2s+1)-manifolds, Topology, 6 (1967), 273-296. 